\section{TRAUMA CARE}

\section{Trauma care-a vision for the future}

For want of trauma surgery as a specialty in the UK, the management of major trauma has come to be seen as the responsibility of specialists in accident and emergency (A\&E) medicine. The management of major trauma perhaps attracts more trainees than any other aspect of the specialty. Consultants all over the country struggle to establish multidisciplinary "trauma teams" and the competition for Advanced Trauma Life Support (ATLS) course places leads to long waiting lists. Yet despite all this interest and activity, the management of the victim of trauma remains suboptimal in many hospitals.

Perhaps the main reason for this is lack of communication between specialties. We have all sat in coffee rooms at antisocial hours and bemoaned the attitude of one or other specialty regarding their response to a particular patient. Yet in many centres, and indeed nationally, there is no culture of specialties training together, learning together, and working together as equals to improve the outcome for trauma patients. It is very easy for specialties, each recognised as excellent in their own field, to contribute to suboptimal overall management when working together.

Apart from promoting the best evidence based resuscitation, $A \& E$ specialists are ideally placed to promote integrated trauma care. As we have all realised, the optimal management of a patient is more than the optimal management of a patient's individual injuries.

The organisation "Trauma Care" was founded in 1996 to promote just such integrated trauma care. After a successful launch conference in Harrogate in 1997, which was designed to promote the philosophy of integrated, multidisciplinary care, the organisation has grown and now represents all the medical specialties involved in trauma care as well as members of specialties allied to medicine such as nurses, paramedics, and scientists.

The abstracts published below are from our most recent conference in Bournemouth. Four hundred delegates attended and the conference was designed to encourage doctors, nurses, and paramedics to participate. The organisation continues to work towards establishing and promoting multispecialty, multidisciplinary evidence based best practice.

The review journal Trauma was launched successfully in 1998 and, in 2000, Trauma Care expert working groups will issue evidence based guidelines for best practice in a number of clinical areas. Also in 2000, the conference Trauma 2000 will see the launch of a new manual of trauma care based on the best of European practice.

Trauma Care is currently the only organisation working in the field of trauma that truly represents all specialties and disciplines, and we are delighted that interest in setting up a similar body has been expressed by leading trauma clinicians in a number of other countries. Our sister organisation Trauma Care (South Africa) is currently being established.

In the UK, we intend to develop a wide range of initiatives including educational projects and the promotion of research and best practice. A research and development committee has recently been formed. We will in due course be moving from a committee based organisation to an open membership with Trauma as our society journal.

May I encourage you to join us in London for Trauma 2000, and to contribute to Trauma Care. The future of this organisation is bright and the opportunities great but in order to work towards the best possible outcome for trauma victims in this country and abroad, we need your support. If you would like more information about the London conference, please contact the Conference Secretariat at Index Communications Meetings Services, Crown House, 28 Winchester Road, Romsey, Hampshire SO51 8AA.

IAN GREAVES

Consultant in Emergency Medicine, British Army, Accident and Emergency Department, Peterborough District Hospital, Thorpe Road, Peterborough PE3 6DA

\section{ABSTRACTS}

\section{2nd Trauma Care Conference: Improving Trauma Care, Bournemouth, 7-9 June 1999}

\section{Oral presentations}

Amputations at the Royal London Hospital 1852-57

\section{E Chaloner}

Leonard Cheshire Department of Conflict Recovery, University College, London

A retrospective analysis of amputations from the archive records of the Royal London Hospital, Whitechapel.

During the period studied, amputations accounted for $34 \%(142 / 400)$ of all operations performed in the hospital. There were 44 upper limb ( 25 above and 19 below elbow) and 98 lower limb amputations ( 51 above and 47 below knee) on 121 males (mean age 29 years; range 1-60) and 15 females with a mean age of 31 years (range 2-75). Six men had bilateral lower limb amputations.

Trauma accounted for 35 arm and $50 \mathrm{leg}$ amputations $(60 \%$ of the total). Industrial accidents caused 27 out of 72 incidents where the cause of accident was known. Sailors, labourers, and railway workers were especially prone to falling victim to accidents requiring amputation.

The overall mortality was approximately $50 \%$ (61 deaths, 69 survivors, six unknown). Of the lower limb amputations carried out for trauma, 34 patients died and 16 survived. Of the upper limb amputations, eight died and 28 survived.

The cause of death (where recorded) was commonly shock (11 cases) or sepsis (32 cases). Chloroform was used in 30 of 136 cases. The mortality where chloroform was used for trauma cases was higher (11 deaths out of 17 cases) than in trauma cases where it was not used ( 23 deaths from 53 cases).

The above data illustrate the high mortality of amputation in the 19 th century. Compared with other contemporary military and civilian figures the mortality is quite high, especially the combination of chloroform and trauma. This may reflect the poor nutritional state of patients in the East End or the effects of anaesthetising patients who were inadequately resuscitated.
The lived experience of resuscitation for accident and emergency nurses $\mathrm{C}$ Williams

Accident and Emergency, West Wales General Hospital, Carmarthenshire

A phenomenological method was used to describe the lived experience of resuscitation for accident and emergency nurses. Twelve nurses were purposefully selected after taking part in a resuscitation. Data was collected from two unstructured interviews with each participant, and then analysed using Colaizzi's (1978) methodology. Findings were written into an exhaustive description of the lived experience, and then returned to the participants for validation.

The lived experience of resuscitation was found to be occasionally positive, producing feelings of achievement and pleasure, due on most occasions to perceptions of teamwork and support from competent colleagues. On other occasions the lived experience engendered feelings of anxiety, distress, frustration, horror, sadness, and anger caused by organisational factors as well as those intrinsic to nursing critically ill patients. Death, if it occurred, was found to come with a period of unacknowledged awareness before a consensus decision was made to end the resuscitation. Death that was perceived as inevitable caused feelings of sadness; death that was unexpected or unaccepted caused intense distress. Talking to relatives after the death was associated with feelings of acute anxiety.

Many staff displayed some signs of stress after a resuscitation, but good social support systems within the workplace enabled the experience, although not forgotten, to be accepted and integrated into the nurses' life experiences within a short period of time.

Recommendations were made to improve organisational factors within resuscitations, increase education for staff in the care of bereaved relatives, and to encourage and sustain the informal social support system already in place.

\section{Emergency management of toxic trauma} D Baker

SAMU 75, Paris, France

Toxic trauma may be defined as the effects on casualties after the accidental or deliberate release of toxic substances. Such a release may occur in both peace and war and may be unexpected. Emergency medical responders should therefore be aware of the problems of toxic release, of the dangers of working in a contaminated environment, and of the special applications of site safety required. They should also be aware of the immediate measures necessary for effective medical management.

Toxic agents cause multisystem dysfunction but the effects on the respiratory system may be life threatening. Recent developments in equipment and training allow the provision of protocols for the provision of advanced life support for toxic trauma within a contaminated environment (TOXALS) and can overcome potentially life threatening delays in the provision of medical care.

Ventilatory support in toxic trauma is vital and is now available using a portable stand alone automatic ventilator, developed originally from a military specification, and which has been produced for both military and civil use. The rationale and practicalities of the use of advanced life support equipment in a toxic zone will be presented within the framework 